\title{
Real time multi-modal super- resolution microscopy through Super- Resolution Radial Fluctuations (SRRF-Stream)
}

Justin Cooper, Mark Browne, Hugh Gribben, Martin Catney, Colin Coates, et al.

Justin Cooper, Mark Browne, Hugh Gribben, Martin Catney, Colin Coates, Alan Mullan, Geraint Wilde, Ricardo Henriques, "Real time multi-modal superresolution microscopy through Super-Resolution Radial Fluctuations (SRRFStream)," Proc. SPIE 10884, Single Molecule Spectroscopy and Superresolution Imaging XII, 1088418 (22 February 2019); doi: 10.1117/12.2510761 


\title{
Real Time Multi-modal Super-Resolution Microscopy through Super- Resolution Radial Fluctuations (SRRF-Stream)
}

\author{
Justin Cooper $^{1 *}$, Mark Browne ${ }^{1}$, Hugh Gribben ${ }^{1}$, Martin Catney ${ }^{1}$, Colin Coates ${ }^{1}$, Alan Mullan ${ }^{1}$, \\ Geraint Wilde ${ }^{1}$, Ricardo Henriques $;{ }^{2}{ }^{1}$ Andor Technology Belfast, UK, ${ }^{2}$ University College London, \\ UK
}

\begin{abstract}
Super-resolution radial fluctuations (SRRF) is a combination of temporal fluctuation analysis and localization microscopy. One of the key differences between SRRF and other super-resolution methods is its applicability to live-cell dynamics because it functions across a very wide range of fluorophore densities and excitation powers. SRRF is applied to data from imaging modes which include widefield, TIRF and confocal, where short frame bursts (e.g. 50 frames) can be processed to deliver spatial resolution enhancements similar to or better than structured illumination microscopy (SIM). On the other hand, with sparse data e.g. stochastic optical reconstruction microscopy (STORM), SRRF can deliver resolution similar to Gaussian fitting localization methods. Thus, SRRF could provide a route to super-resolution without the need for specialized optical hardware, exotic probes or very high-power densities. We present a fast GPUbased SRRF algorithm termed "SRRF-Stream" and apply it to imagery from an iXon EMCCD coupled to a multi-modal imaging platform, Dragonfly. The new implementation is $>300$ times faster than the standard CPU version running on an Intel Xeon $3.5 \mathrm{GHz} 4$ core processor, and > 20 times faster than the NanoJ GPU implementation, while also being integrated with acquisition for real time use. In this paper we explore the image resolution and quality with EMCCD and sCMOS cameras and various fluorophores including fluorescent proteins and organic dyes.
\end{abstract}

\section{Introduction}

In recent years several super-resolution microscopy methods have been developed that allow measurements below the diffraction limit enabling the study of cell structure and processes that weren't previously possible. One of these methods, super-resolution radial fluctuations (SRRF - pronounced 'Surf') is a combination of temporal fluctuation analysis and localization microscopy. One of the key differences between SRRF and other super-resolution methods is that it is highly compatible with imaging the dynamics of live cells. This is as a result of it being used with common fluorophores, over a broad range of densities and excitation powers. The SRRF algorithm was developed by the Henriques group at UCL [2]. This SRRF approach is based on the analysis of the radial and temporal fluorescence intensity fluctuations across a sequence of images. The number of 'input' images per sequence can be adjusted to suit either spatial or temporal resolution. This is especially important for live cell imaging studies where the minimization of phototoxicity is a key concern and if faster, more dynamic physiological processes are under study.

SRRF uses the concept of Radiality Fields [3] as the first important step towards achieving super-resolution. This approach essentially analyses intensity gradients across a user-defined set of radials, intersecting the center point of analysis. The radial gradients are analyzed across every point in the image, usually with an interpolated user-defined resolution that is greater than the native pixel resolution of the acquiring camera. This results in a highly complex field of data that contains rich information on local intensity gradients. Finally, this allows detailed information on the accurate location of fluorophores within the individual image to be derived. 

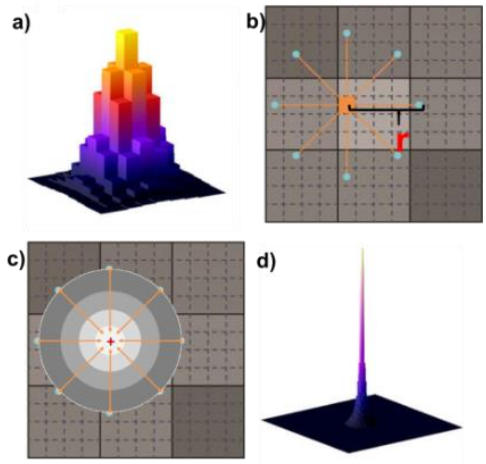

Fig 1. Resolution enhancement by Spatial Analysis - Radiality Transform.

a. A 3D surface plot of diffraction limited Point Spread Function (PSF)

b. $\quad$ The Image is subdivided into subpixel regions (Radiality magnification)

c. The Radiality is calculated for each pixel by calculating intensity gradients at each of 8 points at a radius $r$ from the pixel of interest (Ring Radius). (Real point sources exhibit radially symmetric intensity gradients which converge at the origin).

d. The Radiality at each subpixel shown plotted as a 3D surface plot

The SRRF process requires multiple images (typically 50-100) to be acquired in order for a single super resolved output image to be produced. Stochastic Fluctuation Analysis (SFA) methods, such as SRRF and Super-resolution Optical Fluctuation Imaging (SOFI) [2], are dependent on the analysis of (short) image time series, with relatively low exposures and fast sampling. The value assigned to an output pixel depends on the original brightness and the correlation of fluorescence fluctuations for that pixel. Since the background tends to be poorly correlated, gains in image contrast can also be achieved.

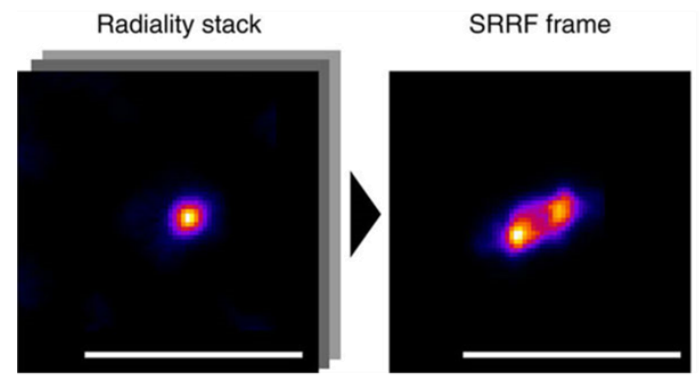

Fig 2. Resolution enhancement by temporal fluctuations. A time series of radiality maps can be used to further enhance the spatial localization by statistical analysis of the temporal fluctuations. Temporal radiality maxima or average projections produce a single super-resolution SRRF frame from a radiality stack.

For SOFI, improvements in resolution can be derived from computing higher order cumulants. In the case of SRRF, resolution increases are achieved from continuous interpolation of the radiality field. SFA methods are able to handle high fluorophore densities, making SRRF applicable to the common microcopy techniques: widefield, confocal and TIRF images with relatively low excitation intensities. In this paper we discuss a development of SRRF termed "SRRFStream" which enables real time live cell super-resolution as opposed to post processing. SRRF is available as a free open-source plugin for ImageJ or Fiji image analysis software. SRRF-Stream is available solely through Andor iXon EMCCD and Sona sCMOS cameras.

\section{Materials and Methods}

In order to access SRRF-Stream requirements are as follows, Camera: A SRRF-Stream compatible iXon Life or iXon Ultra EMCCD or Sona sCMOS camera, SRRF-Stream license and installer (enables SRRF-Stream in camera - specific to camera serial number). A CUDA-compatible NVidia GPU card (the Nvidia GPU card should have Compute Capability v3.0 or above and 4GB or greater on-board GPU RAM). Software: MicroManager (64-bit) software, Andor 
Fusion Software or Andor SDK2. Microscope: A fluorescence microscope with widefield, TIRF or spinning disk confocal modality.

\section{Results and Discussion}

\section{Optimization of GPU Processing}

The main difference between SRRF and SRRF-Stream is the optimization of the SRRF algorithm processing and execution. By optimizing the GPU processing techniques, the SRRF algorithm implemented in SRRF-Stream, runs up to 30x faster than the existing ImageJ-based post processing implementation of SRRF ('NanoJ-SRRF'), using the same GPU card (Fig 3). This significant acceleration enables workflow enhancement, by further allowing data acquisition and SRRF processing to operate in parallel. As soon as the first input image is transferred to the GPU card, the processing begins; SRRF-Stream does not wait until the full image sequence has been recorded before beginning the processing. Since processing is significantly faster than the camera image acquisition process (typically 6 - 9x faster than even 'overclocked' iXon EMCCDs), SRRF-Stream is able to provide real time super-resolution, with large field of view super resolution images. An EMCCD full field of view super-resolved image can be readily generated at a rate of approximately $1 \mathrm{fps}$, up to > 10 fps using smaller standard or Image Crop ROIs.

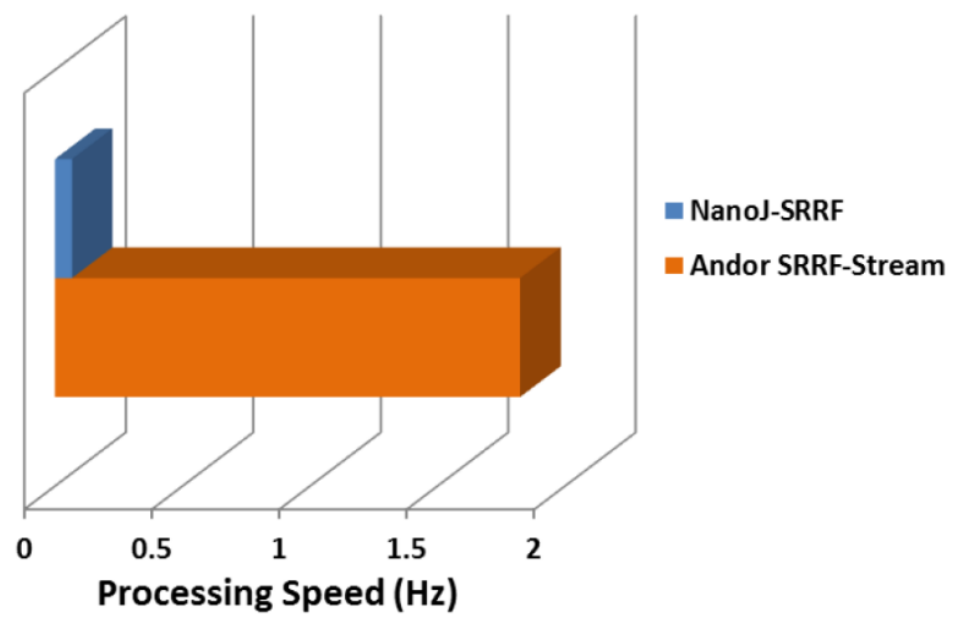

Fig 3. A comparison of NanoJ-SRRF and Andor SRRF-Stream which has been optimised for increased processing speed. This graph compares the rate of processing of blocks of 100 raw input images (1024 x 1024 pixels), to yield resultant SRRF super-resolution images of $4096 \times 4096$ pixels. SRRF-Stream is compared to NanoJ-SRRF, the processing occurring on the same Nvidia GTX 1070 GPU card. The SRRF-Stream acceleration subsequently allows data acquisition and processing to happen in parallel, yielding a further workflow improvement over NanoJ-SRRF.

\section{The importance of speed for live cell Imaging - SRRF-Stream frame rate performance}

Some live cell processes, such as mitosis, can be followed adequately using time-lapse imaging and the camera can readily be operated in full pixel resolution coupled with optimal settings for achieving best super-resolution, whilst maintaining necessary frame rate. Even when combining multi-colour and z-stack time-lapse super-resolution imaging, it is possible to observe relatively large fields of view.

However, some physiological processes are dynamic and therefore require multiple SRRF-output frames per second. As the temporal resolution is of SRRF-Stream is limited by the camera, some compromise must be made to the field of view and/or the number of input frames per output SRRF image, in order to increase the frame rate.

For the iXon EMCCD camera series, a feature called 'Crop Mode', in conjunction with Region of Interest, yields significant frame rate increases. Crop Mode essentially 'fools' the sensor into thinking it is smaller than it actually is, and thus it ignores the need to readout and dump charge from the pixels that surround the specified ROI. In order to use 
Crop Mode a simple accessory called 'OptoMask' is used to optically shield regions outside of the defined ROI from photons which would otherwise distort the image.

Table 1 shows SRRF-Stream super-resolution frame rates achievable for the iXon 888 model for different combinations of ROI size (Standard ROI and Crop Mode ROI) and input frames per super-resolved output image. For example, a 128 x 128 ROI of the iXon 888, used in Crop Mode, and inputting 50 frames per output image (thus sacrificing some degree of resolving power) would output $14 \mathrm{fps}$. This is more than adequate for following many live cell processes. Note also that if a 4x magnification is used in SRRF-Stream (as is typical), a 128 x 128 ROI becomes a 512 x 512 super-resolution image.

\begin{tabular}{|c|c|c|c|}
\hline \multicolumn{4}{|c|}{ Standard ROI Mode } \\
\hline iXon 888 ROI Size & $\begin{array}{l}\text { Camera Frame rate - } \\
\text { Standard ROI (fps) }\end{array}$ & $\begin{array}{l}\text { SRRF-Stream Frame rate } \\
\text {-100 input frames (fps) }\end{array}$ & $\begin{array}{l}\text { SRRF-Stream Frame } \\
\text { rate- } 50 \text { input frames } \\
\text { (fps) }\end{array}$ \\
\hline $512 \times 512$ & 50 & 0.5 & 1.0 \\
\hline $256 \times 256$ & 95 & 1.0 & 1.9 \\
\hline $128 \times 128$ & 171 & 1.7 & 3.4 \\
\hline \multicolumn{4}{|c|}{ Crop Mode ROI Mode } \\
\hline & $\begin{array}{l}\text { Camera Frame rate - } \\
\text { Crop Mode ROI ( fps) }\end{array}$ & $\begin{array}{l}\text { SRRF-Stream Frame rate } \\
\text {-100 input frames (fps) }\end{array}$ & $\begin{array}{l}\text { SRRF-Stream Frame } \\
\text { rate- } 50 \text { input frames } \\
\text { (fps) }\end{array}$ \\
\hline $512 \times 512$ & 78 & 0.8 & 1.6 \\
\hline $256 \times 256$ & 251 & 2.5 & 5.0 \\
\hline $128 \times 128$ & 697 & 7 & 14 \\
\hline
\end{tabular}

Table 1 - SRRF-Stream super-resolution frame rates achievable for the iXon 888 model for different combinations of ROI size (Standard ROI and Crop Mode ROI) and input frames per super-resolved output image.

The underlying SRRF algorithm is the same whether running on data acquired from Sona SCMOS or an iXon EMCCD camera, thus the frame-rate performance on a ROI of identical size with the same parameters (for SRRF processing only) should be the same. The main factors in the speed of the SRRF algorithm are:

1. The size of the raw input data (this is approximately linear in XY, i.e. a frame of size $1024 \times 1024$ take 4 times longer to process than a frame of size $512 \times 512$ )

2. The SRRF Frame Count

3. The SRRF Radiality Magnification (a magnification of $M$ takes $M / N^{\wedge} 2$ times longer than a magnification of $\mathrm{N})$.

4. The CUDA enables graphics card used for processing - higher performance cards will process the data more quickly.

\section{Optimal imaging criteria for SRRF-Stream}

SRRF delivers optimal image quality if the initial images are acquired at, or close to, Nyquist conditions. This is the case for all modalities. For relatively large pixel cameras such as iXon 897 or iXon 888 models, this typically would involve additional magnification in the form of a tube lens in the microscope, or from a C-mount coupler with built-in magnification. Some example combinations of Objective Mag and Coupler Mag are shown in Table 2, showing the range of oversampling of the resolution limit. The ideal oversampling is according to Nyquist is 2.3 or greater, but note that conditions down to 1.5 can still adequately be used to yield good results with the SRRF algorithm. 


\begin{tabular}{|l|l|l|l|l|l|l|l|}
\hline & $\begin{array}{l}\text { Region of } \\
\text { Interest }\end{array}$ & $\begin{array}{l}\text { Pixel } \\
\text { Size } \\
(\mu \mathrm{m})\end{array}$ & $\begin{array}{l}\text { Objective } \\
\text { Mag }(x)\end{array}$ & NA & $\begin{array}{l}\text { Coupler } \\
\text { Mag }(x)\end{array}$ & $\begin{array}{l}\text { Resolution } \\
\text { Limit on } \\
\text { Sensor- } \\
\text { Rayleigh } \\
(\mu m)\end{array}$ & $\begin{array}{l}\text { Nyquist } \\
\text { Sampling } \\
\text { (Res limit } \\
\div \text { pixel } \\
\text { size) }\end{array}$ \\
\hline \multirow{3}{*}{$\begin{array}{l}\text { iXon } \\
897\end{array}$} & $512 \times 512$ & 16 & 100 & 1.49 & 1 & 24.56 & 1.5 \\
\cline { 2 - 8 } & $512 \times 512$ & 16 & 100 & 1.49 & 1.5 & 36.85 & 2.3 \\
\cline { 2 - 8 } & $512 \times 512$ & 16 & 60 & 1.4 & 1.5 & 23.53 & 1.5 \\
\hline \multirow{3}{*}{$\begin{array}{l}\text { iXon } \\
888\end{array}$} & $1024 \times 1024$ & 13 & 100 & 1.49 & 1 & 24.56 & 1.9 \\
\cline { 2 - 8 } & $1024 \times 1024$ & 13 & 100 & 1.49 & 1.5 & 36.85 & 2.8 \\
\cline { 2 - 8 } & $1024 \times 1024$ & 13 & 60 & 1.4 & 1.5 & 23.53 & 1.8 \\
\cline { 2 - 8 } & $1024 \times 1024$ & 13 & 60 & 1.4 & 2.0 & 31.37 & 2.4 \\
\hline
\end{tabular}

Table 2 - Example combinations of Objective Mag and Coupler Mag, resulting in varying degrees of oversampling

It should be noted that like all super-resolution techniques, occasionally SRRF-Stream may result in certain image artefacts. These may be observed as:

1) A 'Shadow region' surrounding cellular structures or,

2) 'Star-shaped' patterning in the image when zoomed in

The shadowing effect is caused by the SRRF algorithm and the way it deals with intensity information contained within the original image. The algorithm looks at localised area around a visible feature, to identify the location of fluorophores by their high local radiality. It is important to recognize that this shadowing effect will only ever manifest in true background, thus would not adversely affect the quantitative integrity of the data. For example, if there were two, side by side closely situated objects e.g. microtubules then one would not be affected by a contrast shadow of the other, as the SRRF algorithm would simply have not detected contrast information to produce the shadow effect.

A star-shaped pattern artefact may become apparent when your setup has not been optimized for lens magnification, NA and camera pixel size and is, as a result, under-sampling. Therefore, the microscope set-up should be configured as described above to ensure Nyquist sampling is achieved. It is also possible to increase the ring-radius setting to reduce this type of artefact, at the expense of resolution.

\section{Comparing SRRF-Stream with other techniques under a range of different imaging modalities}

SRRF is readily applied to data from imaging modalities such as widefield, confocal and TIRF, where short frame bursts (e.g. 50-100 frames) can be processed to provide spatial resolution equal to, or better than that of structured illumination microscopy (SIM) [4]. The resolution that can be achieved by SRRF-Stream is typically between $50-150 \mathrm{~nm}$.

\section{Application of SRRF-Stream to Widefield Fluorescence}

A comparison of a widefield fluorescence image with and without SRRF-Stream applied is shown in Fig 4. In order to provide a comparable relative image, without SRRF-Stream, 100 standard widefield images were acquired and then averaged. While the original image was of a larger field of cells, a zoomed ROI of one cell is shown in order to more easily display a line intensity profile comparison through a small region. The improvement in resolving power of SRRFStream is readily apparent. 


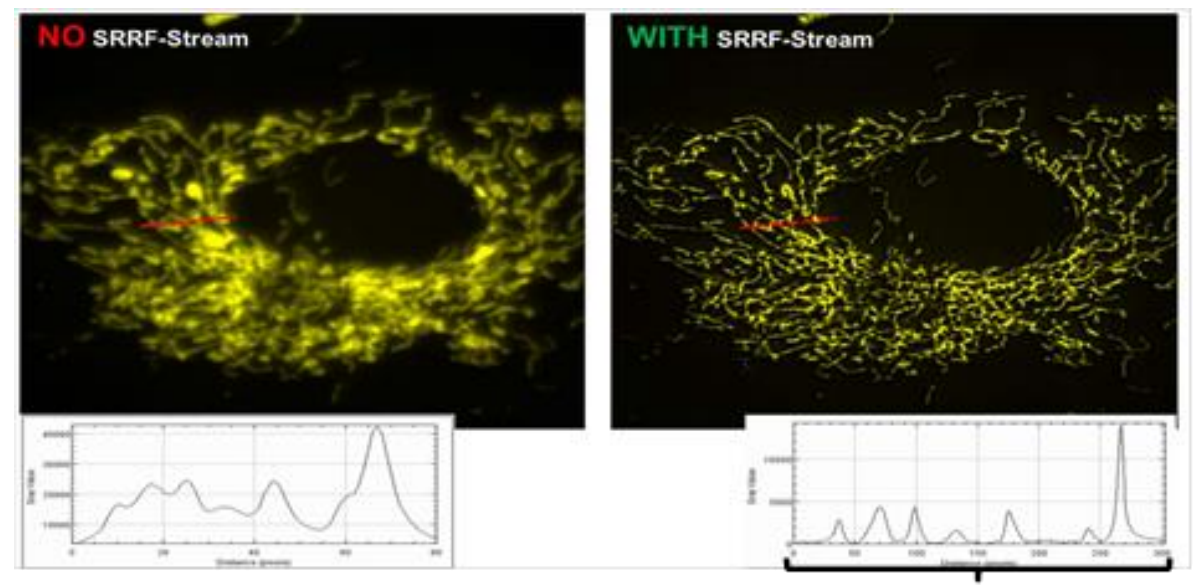

Fig 4. Image comparison of a fluorescently labelled BPAE cell, recorded with a widefield fluorescence microscope and a SRRFStream enabled iXon Life 888 EMCCD camera.

a. Wide-field image of BPAE cell: Avg. 100 images, $50 \mathrm{~Hz}$ readout rate, $\mathrm{FWHM} \sim 1 \mu \mathrm{m}$

b. SRRF rendered image: Mag. 4x, Ring radius 0.5, 100 input images for temporal analysis, $0.5 \mathrm{~Hz}$ super-resolution readout rate, $F W H M \sim 200 \mathrm{~nm}$

A further illustration of the improved resolution for widefield fluorescence is shown in Fig 5. Again, an improvement in resolution can be seen in the SRRF-Stream images (5b and 5c) when compared to the equivalent image without SRRFStream applied (5a) which has a higher FWHM value.
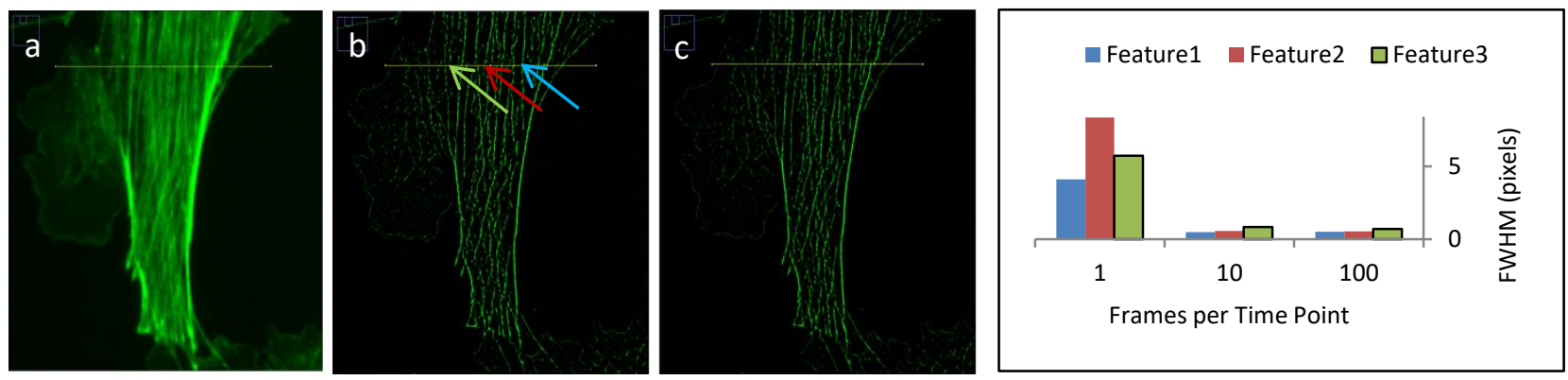

Fig 5. Actin Filaments (BPAE Alexa Fluor 488): a. Widefield - 1 frame/ timepoint, b. SRRF - 10 frames/timepoint, c. SRRF - 100 frames/timepoint.

\section{Application of SRRF-Stream to Confocal Microscopy}

Significant improvements are also observed for confocal microscopy. An example of this is shown in Fig 6 and Fig 7. The improvement in resolving power of the image with SRRF-Stream applied can observed in the level of detail in the mitotic spindle region. The comparative line intensity profile drawn through this region, shows the multiple intensity peaks for the SRRF-Stream image, whereas discrete intensity peaks are not present without SRRF-Stream. SRRF-Stream can be combined with confocal to capture super-resolution deep in thick samples, such as resolving small dendritic spines on nerve cells. 

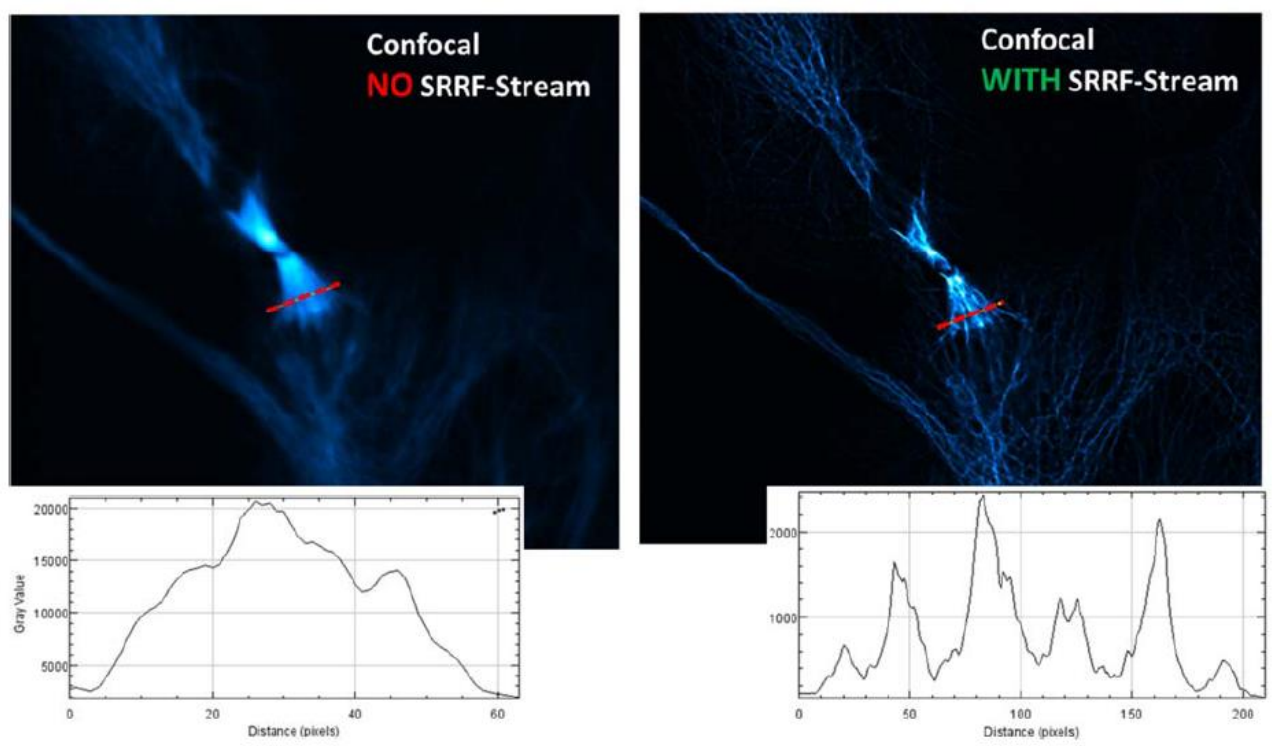

Fig 6. Comparison of a fluorescently labelled U2OS cell line* recorded with an Andor Dragonfly confocal spinning disk fluorescence microscope and a SRRF-Stream enabled iXon Life 888 EMCCD camera. A x63 objective was used, with further $2 x$ magnification and $488 \mathrm{~nm}$ illumination. *U2OS cell line was fixed, stained with anti-alpha-tubulin primary antibody (green, AF488) and phalloidin (red, rhodamine) to visualize F-actin, DAPI staining to visualize nuclei. Samples prepared by Klebanovych A., Laboratory of Biology of Cytoskeleton, IMG of the AS CR, v.v.i.
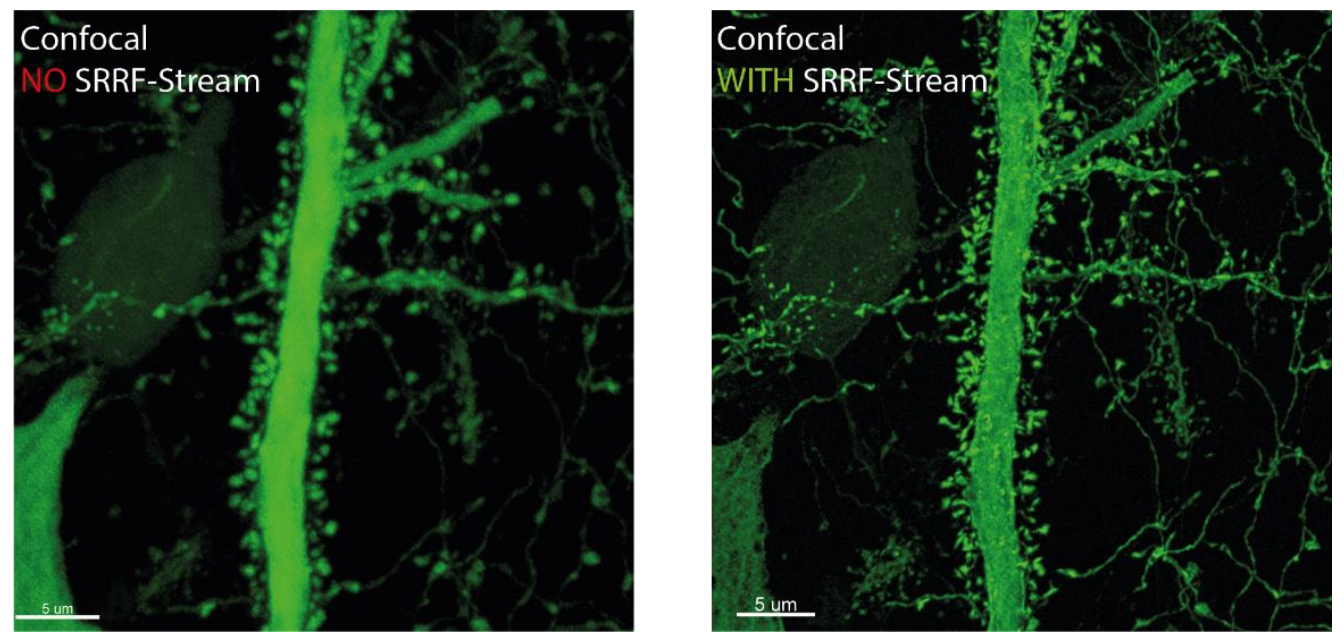

Fig 7: Confocal with SRRF-Stream for Deep super-resolution Imaging. YFP-expressing neurons in cleared mouse brain (200um thick section). 90 slice volume imaged 70 microns into the sample over 11um range. 100x (1.45 NA) objective with 1.6x additional magnification for Nyquist sampling. Each SRRF image per z-plane is comprised 100 raw frames. Sample from SunJin Lab in collaboration with Dr Chia-Ming Lee, Academia Sinica, Taiwan.

\section{Comparison with TIRF}

Total Internal Reflection Fluorescence (TIRF) Microscopy can be used as a means to increase the effective axial resolution or localization accuracy within a restricted region of a thin sample in proximity to the glass coverslip. Therefore, TIRF is typically used to study processes at the plasma membrane such as single protein trafficking or endoor exocytosis that benefit from improved resolution within an optical plane of interest. SRRF-Stream can also provide benefits to these applications, and a comparison of SRRF-Stream and conventional TIRF microscopy is shown in Fig 8. In this example, TIRF images are compared versus TIRF images in SRRF using AlexaFluor 488 labeled Actin. On a 
separate note, Particle Image Velocity (PIV) analysis reveals that flow fields that are not discernible with conventional TIRF can be resolved through use of SRRF-Stream.[1]
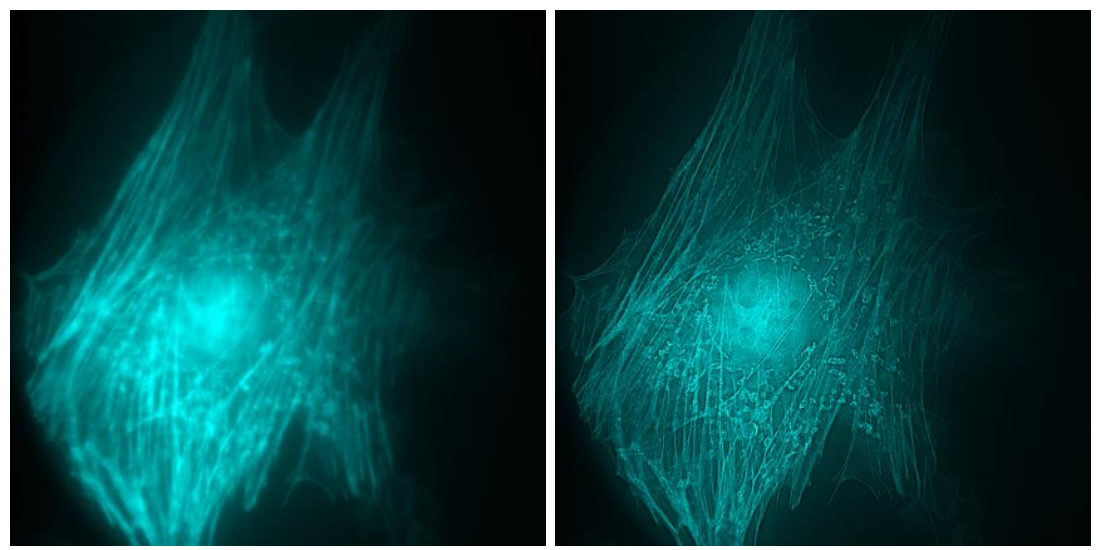

Fig 8. TIRF images (left) are compared versus TIRF and SRRF images (right) of AlexaFluor 488 labeled Actin.

\section{Comparison with SIM}

Structured illumination microscopy (SIM) uses a patterned illumination technique to excite the sample. The subsequent moiré interference patterns provide sub-diffraction limit image information which can be processed to improve image resolution. SIM is theoretically limited to a 2 -fold reduction of the classical diffraction limit. Like SRRF-Stream it is compatible with conventional fluorophores. A comparison of SRRF-Stream with this technique is given in Fig 9. The images are of the same field of cells, recorded on the same microscope, using identical objective and optical path. The only difference being that SIM was recorded using a sCMOS detector with $6.5 \mu \mathrm{m}$ pixels whereas the widefield and resultant SRRF was recorded using an iXon EMCCD detector with $16 \mu \mathrm{m}$ pixels. The superior resolving power of SRRF relative to SIM is evident, indicative that SRRF is achieving a greater than 2 -fold improvement over the classical diffraction limit.
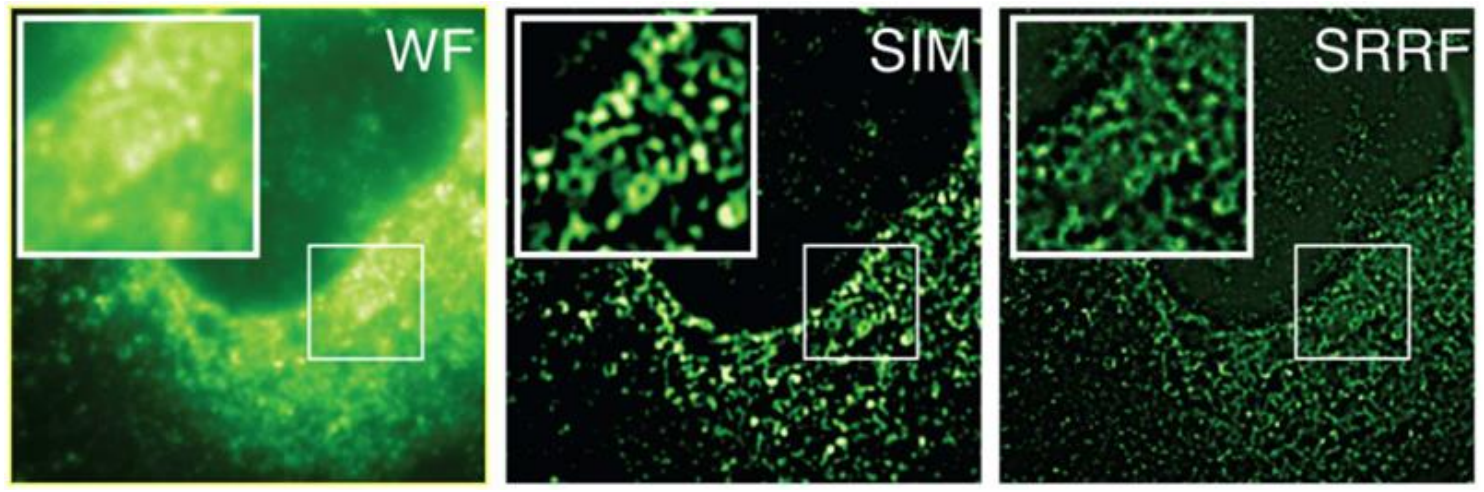

Fig 9. HCV infected cells stained with anti-NS5A. Comparison of Widefield (WF), Structured Illumination Microscopy (SIM) [4] and SRRF images (SRRF of the widefield image). Sample courtesy of the Grove lab at UCL.

\section{Use of SRRF-Stream on Sparse Datasets}

STORM and other super resolution techniques that are based on photoactivated localization microscopy (PALM) are now well established. Such techniques enable lateral resolutions as low as 50 to $30 \mathrm{~nm}$ to be attained [5]. When SRRFStream is used with sparse datasets such as those that are typical of such techniques [6], SRRF provides a resolution, approaching $50 \mathrm{~nm}$. It has been shown that 2 Gaussian PSFs separated by $2 \sigma$ are resolvable after 1 radiality transform. [1] For $1.4 \mathrm{NA}, 60 \mathrm{x}, 550 \mathrm{~nm}$ emission this corresponds to fluorophore centers < $160 \mathrm{~nm}$ apart. After temporal analysis has 
been performed, structures with a separation of $<100 \mathrm{~nm}$ are resolvable. It is also important to note that the levels of illumination required for SRRF-Stream are orders of magnitude lower than STORM. This means that SRRF is more compatible with live cells and ensuring accurate determination of the native cellular processes.

\section{Conclusion}

SRRF provides a highly flexible and adaptable route to super-resolution without the need for specialized optical hardware or exotic probes and utilizing relatively low power densities. Optimization of SRRF GPU processing has enabled a significant increase in algorithm execution and associated tasks in SRRF-Stream. This enables real-time live cell super-resolution to be achieved across a range of image modalities. SRRFStream is a camera based solution, running on Andor iXon EMCCD and Sona SCMOS cameras and requiring a suitable CUDA enabled graphics card. Such a solution is widely applicable to life science research as further information can be derived from structures and processes that were not previously possible.

\section{References.}

1. Gustafsson, N., Culley, S., Ashdown, G., Owen, D. M., Pereira, P. M., and Henriques, R. Fast live-cell conventional fluorophore nanoscopy with ImageJ through super-resolution radial fluctuations. Nature Communications 2016 7(12471):12471.

2. T. Dertinger, R. Colyer, G. Iyer, S. Weiss, and J. Enderlein Fast, background-free, 3D super-resolution optical fluctuation imaging (SOFI) Proceedings of the National Academy of Sciences Dec 2009, 106 (52) 2228722292; DOI: 10.1073/pnas.0907866106

3. Parthasarathy, R. Rapid, accurate particle tracking by calculation of radial symmetry centers. Nat Methods Jun 2012 10;9(7):724-64.

4. Gustafsson, M. G. L., Agard, D. A., and Sedat, J. W. I I M: 3D widefield light microscopy with better than 100nm axial resolution Journal of Microscopy, Vol. 195, Pt 1, July 1999, pp. 10-16

5. Rust, M. J., Bates, M. and Zhuang, X. Sub-diffraction-limit imaging by stochastic optical reconstruction microscopy (STORM). Nat. Methods 3, 793-795 (2006).

6. Huang, B., Wang, W., Bates and M., Zhuang, X. Three-Dimensional Super-Resolution Imaging by Stochastic Optical Reconstruction Microscopy Science 08 Feb 2008 : 810-813. 